\title{
Development of a simultaneous vibration and pressure stimulation system for cognitive studies
}

\author{
Soon-Cheol Chung ${ }^{\mathrm{a}}$, Mi-Hyun Choi ${ }^{\mathrm{a}}$, Sung-Jun Park ${ }^{\mathrm{a}}$, Jung-Chul Lee ${ }^{\mathrm{a}}$, Ul-Ho Jeong ${ }^{\mathrm{a}}$, Ji-Hye \\ Baek $^{\mathrm{a}}$, Ji-Hye You ${ }^{\mathrm{a}}$, Young Chil Choi ${ }^{\mathrm{b}}$, Dae-Woon Lim ${ }^{\mathrm{c}}$, Jeong-Han Yi ${ }^{\mathrm{a}}$ and Hyung-Sik \\ $\mathrm{Kim}^{\mathrm{a}, *}$ \\ ${ }^{a}$ Department of Biomedical Engineering, Research Institute of Biomedical Engineering, College of \\ Biomedical \& Health Science, Konkuk University, Chungju, South Korea \\ ${ }^{b}$ Department of Radiology, School of Medicine, Konkuk University, Chungju, South Korea \\ ${ }^{c}$ Department of Information \& Communication Engineering, Dongguk University, Seoul, South Korea
}

\begin{abstract}
In this study, a tactile stimulator that could separately or simultaneously display the vibrotactile and pressure sense was developed. The developed system consisted of a control unit, a drive unit, and an actuator, and can be operated with PC or manually. This system quantitatively controls the stimulation parameters such as the stimulation intensity, duration, frequency, and stimulation type. A preliminary electroencephalogram (EEG) experiment for three types of stimulation (vibrotactile, pressure sense, vibrotactile + pressure sense) highlights that the system could be used in complex tactile cognitive studies. An event-related desynchronization (ERD) and synchronization (ERS) were measured at the area of C3 and $\mathrm{C} 4$ for all three types of stimulation, and a clear response was identified in the contralateral somatosensory area from the brain topology. Therefore, it is expected that this system could be widely used in single and complex human tactile cognition and perception studies for vibrotactile and pressure sensation.
\end{abstract}

Keywords: Tactile stimulator, vibration, pressure, simultaneous stimulation, electroencephalogram

\section{Introduction}

Recently, many studies have been performed to develop tactile interfaces and to identify the characteristics of human tactile cognition for use in haptic technology for applications such as entertainment, medical, and military [1,2]. Tactile interfaces typically display vibrotactile, pressure, and thermal senses separately. These stimulators have been used to evaluate the characteristics of human cognition in tactile sensation subjectively or objectively with functional magnetic resonance imaging (fMRI) and electroencephalogram (EEG) [3-6]. Also, complex tactile cognitive studies have

\footnotetext{
${ }^{*}$ Corresponding author: Hyung-Sik Kim, Department of Biomedical Engineering, Research Institute of Biomedical Engineering, College of Biomedical \& Health Science, Konkuk University, Chungju, South Korea. Tel.: +82-10-3309-3302; Fax: +82-43-851-0620; E-mail: hskim98@kku.ac.kr.
} 
emerged to make use of the recently developed system that could simultaneously display vibrotactile and thermal sense [7].

Methods to display vibrotactile stimulation use a piezo element, bimetal, DC motor, servo motor, and so on [8-13]. Methods of stimulating pressure sense use compressed air, cuff, von-frey filament, etc. $[14,15]$. These conventional methods of displaying vibrotactile and pressure sense have various disadvantages with respect to the quantitative control of stimulation parameters such as the stimulation frequency (DC motor, servo motor, bimetal, and cuff), stimulation intensity (piezo element, bimetal, and von-frey filament), stimulation area (piezo element, bimetal, DC motor, servo motor, and cuff), and time delay (DC motor and cuff) and can display only one type of tactile stimulation at a time. Because most tactile sensation perceived daily by humans are caused by various types of complex as well as separate stimulation, a complex tactile cognitive study with the development of complex tactile stimulation interface and a method to overcome limitations of the previously developed stimulators are needed.

This study is intended to develop a tactile stimulator that can separately or simultaneously display vibrotactile and pressure sense to induce complex and separate tactile sensations. Stimulation parameters such as the stimulation frequency, intensity, and duration were controlled quantitatively with a micro-controller. The operation status of the developed system was tested with respect to the control of the stimulation parameters. A preliminary electroencephalogram (EEG) experiment with three types of stimulations (vibrotactile, pressure sense, vibrotactile + pressure sense) demonstrates that the developed tactile stimulation system could be used for complex human tactile cognitive studies.

\section{Methods}

Figure 1 shows a block diagram of the vibration- and pressure-stimulation system. The developed system consists of three parts; a control unit, a drive unit, and an actuator. An electric coil was used for

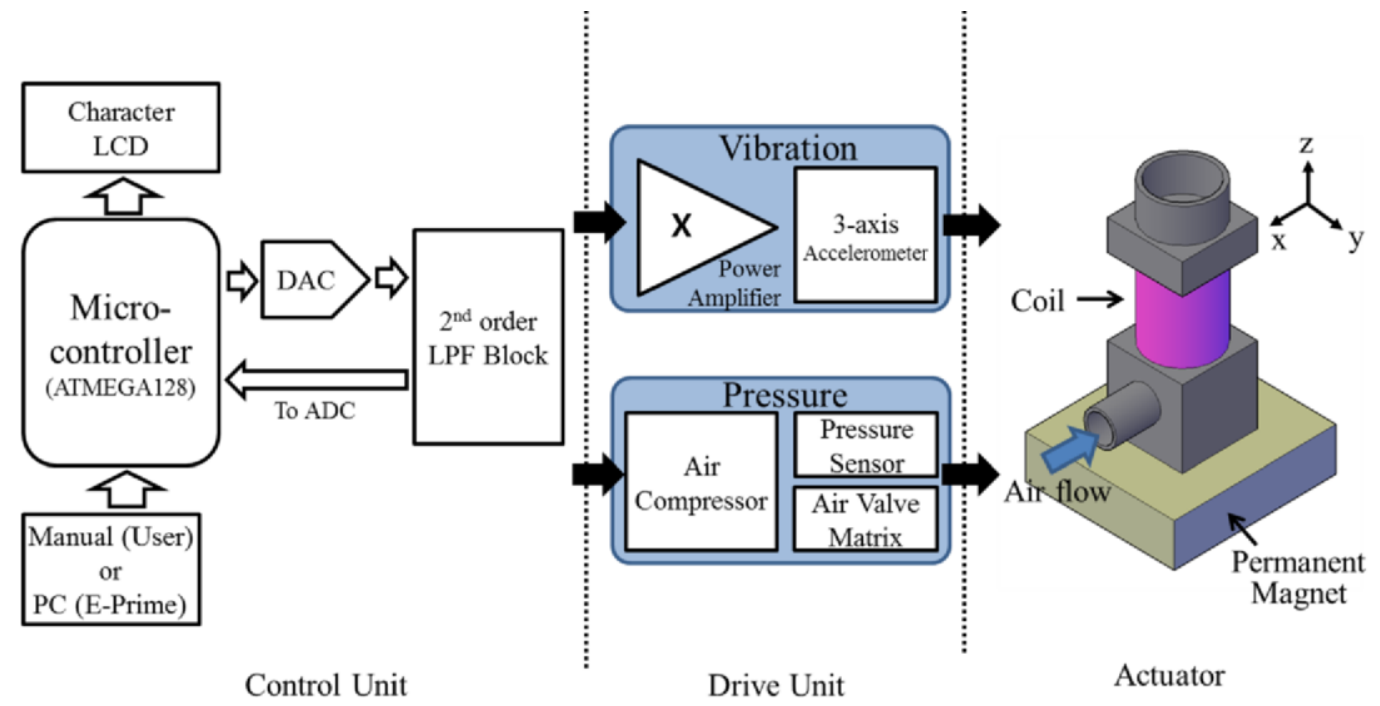

Fig. 1. Block diagram of the vibration- and pressure-stimulation system. 
vibrotactile stimulation [8], and an air compressor was used to stimulate pressure sense. The system can be controlled manually or with a PC equipped with E-Prime software (Psychology Software Tools Inc., USA). E-Prime is a software tool for presenting a visual and an auditory stimulation for use in psychological research, neuroscience study, as well as cognitive and behavioral experiments $[16,17]$.

\subsection{Control unit}

The control unit uses an AVR-family ATMEGA 128 controller (ATMEL, USA), which is a low power and high-performance 8-bit micro-controller. This controller operates at a 4.5 to 5.5 working voltage, and it has several function blocks, such as four 8- and 16-bits timer/counters, comparator, communication interfaces, eight-channel 10-bit analog-to-digital converter and programmable input/output lines. The stimulation waveform of sine wave for vibration was generated with 12-bit digital-to-analog converter AD7545A (Analog Device, USA). The digital noise of the DAC was reduced using the 2'nd order low-pass filter (LPF). The filtered sinusoidal wave is transmitted to the power amplifier of the drive unit. Vibrotactile stimulation can be performed at 18 levels of intensity ( 0 to 9.0 gravity), 40 levels of frequency ( 0 to $400 \mathrm{~Hz}$ ), and time in the micro-seconds ranges. Pressure is controlled by the duty rate of the pulse width modulation (PWM) regulated by the micro-controller timer/counter. This signal is sent to the air compressor of the drive unit. Pressure stimulation can be presented at 18 levels of intensity ( 0 to $9 \mathrm{psi}$ ) and time in seconds. The stimulation parameters such as frequency, intensity, and duration were controlled in real-time by measuring the vibration signals from a 3-axis accelerometer MMA7260Q (Freescale Semiconductor, USA), which was placed on the upper side of the actuator, and the pressure stimulation signal was obtained from a pressure sensor 33A005G-2210 (Smate, Taiwan), which was positioned between the air compressor and the actuator.

\subsection{Drive unit}

The drive unit was responsible for generating compressed air and supplying electrical power to operate the actuator in response to commands from the control unit. A block to drive the vibrotactile stimulation was designed to supply sufficient electric power to the vibration coil after amplifying the sinusoidal signal received from the control unit through a 20-watts audio power amplifier LM1875 (National Semiconductor, USA). A pressure sense driving block controlled the air flow by adjusting the air valve matrix, which was composed of three valves, an air regulator, and an air compressor (JS20, Join Medical Co., Korea) according to the PWM signal sent from the control unit.

\subsection{Actuator}

The actuator was designed to display the two types of tactile stimulation separately or simultaneously without changing the actuator. The outward shape was designed using the 3dimensional computer-aided design and drafting software AUTOCAD (AutoDesk, USA) and a 3-D printing process was used to print a prototype of the actuator with a Mojo 3-D printing system (Stratsys, USA) (Figure 2). The electric coil to generate vibration was wound at the center of the actuator in the shape of a solenoid using copper wire with a diameter of $0.3 \mathrm{~mm}$. A three-axis accelerometer to monitor the vibration was positioned on top of the actuator that was closest to the finger. A neodymium permanent magnet was used to generate the external magnetic field $\left(\mathrm{B}_{0}\right)$ necessary for vibration of coil. The air compressor for displaying pressure sense was connected to the actuator through a poly vinyl chloride (PVC) air tube with an inner diameter of $8 \mathrm{~mm}$. A circular 


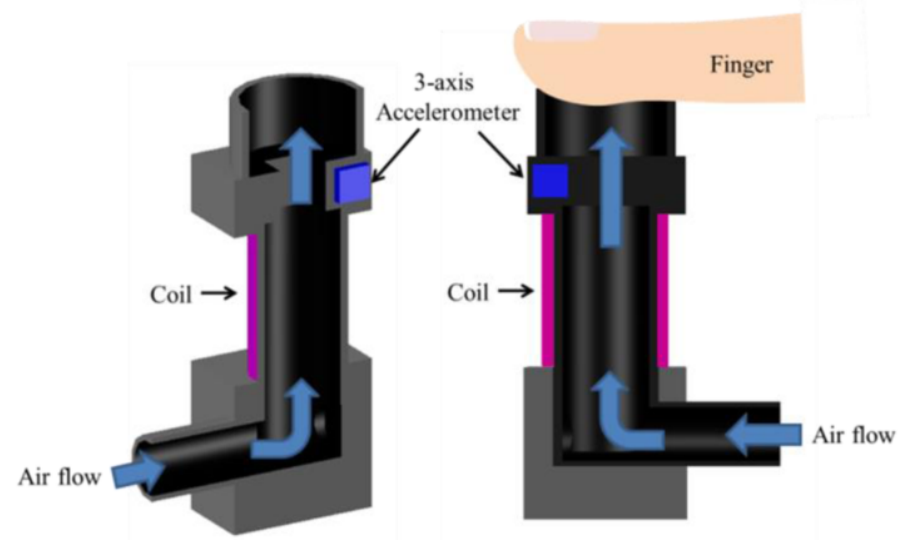

Fig. 2. 3-D CAD design of the vibration- and pressure-stimulation system.

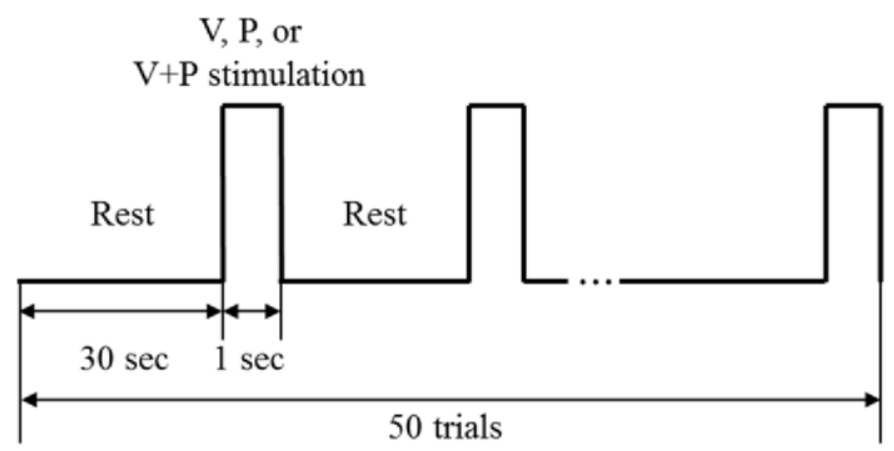

Fig. 3. Experimental design. Three types of tactile stimulation were used; vibration (V), pressure (P), or vibration + pressure $(\mathrm{V}+\mathrm{P})$ stimulation.

channel with a diameter of $6 \mathrm{~mm}$ was constructed inside the actuator so that the air would flow uniformly.

\section{Experiments}

The performance of the developed system was tested. All 40 levels of vibrotactile stimulation frequencies and intensities and 18 levels of pressure sense stimulation were examined. The four analog signals of three-axis accelerometer ( 3 channels) and pressure sensor (1 channel) were simultaneously recorded with a TDS3034 (Tektronix, USA) four-channel digital oscilloscope (sampling rate was $5,000 \mathrm{samples} / \mathrm{sec}$ ). All three types of stimulation signal (vibrotactile, pressure sense, vibrotactile + pressure sense) were stimulated on the distal phalanx of the index finger respectively.

A preliminary EEG experiment on 15 healthy males and 4 females with an average age of $22.6 \pm 2.2$ years was performed in order to verify the developed system. EEG data was recorded at a sampling frequency of $500 \mathrm{~Hz}$ using Quick Amp (Brain Product, Inc, USA). The EEG was measured using an $\mathrm{Ag} / \mathrm{AgCl}$ electrode at 16 areas (AF3, AF4, P3, Pz, P4, F1, F2, F5, F6, C3, Cz, C4, CP1, CP2, Pz, and $\mathrm{Oz}$ ) including $\mathrm{C} 3$ and $\mathrm{C} 4$ according to the 10-20 international electrode system. Figure 3 shows the 
experimental paradigm. Each subject participated in all three types of experiments including stimulation of vibrotactile and pressure senses independently, as well as stimulation of both senses simultaneously.

The experiment began when the EEG was stabilized while the subject sat comfortably in a chair. One of the three types of stimulations (vibrotactile stimulation, pressure sense stimulation, or vibrotactile stimulation + pressure sense stimulation) was applied to the distal phalanx of the subject's right index finger 50 times. The stimulation duration was $1 \mathrm{sec}$, and the resting time between stimulations was $30 \mathrm{sec}$. Experiments for the remaining two types of stimulations were executed in the same fashion. Stimulations were displayed in a random order to remove repetition and carryover effects. The vibrotactile stimulation was 1.8 gravity in stimulation intensity and $200 \mathrm{~Hz}$ in stimulation frequency. The pressure sense stimulation was 9 psi in intensity. It was impossible for the subject to know which stimulation type was stimulated because of the presence of a blackout curtain. The acquired EEG signal was band-pass filtered at 0.5 to $40 \mathrm{~Hz}$ by using MATLAB software (Mathworks, USA). The raw data was analyzed in $2.5 \mathrm{sec}$ intervals divided into $0.5 \mathrm{sec}$ before stimulation, $1 \mathrm{sec}$ of stimulation, and $1 \mathrm{sec}$ after stimulation. Spectrogram and topography analysis was performed over the somatosensory area including $\mathrm{C} 3$ and $\mathrm{C} 4$.

\section{Results}

Figure 4 is an actual picture of the developed system. The main supply voltage to operate the entire system was +12 direct current (DC) volts and converted to +5 volts for use in control unit and +15 volts to drive the drive unit using step-down and step-up regulators, respectively. The maximum power consumption was 82 watts and measured with electric power analyzer DW-6093 (Lutron Electronics, USA). A long-term test for 2 continuous hours in maximum stimulation intensity for simultaneous vibrotactile and pressure sense was carried out to verify the stable operations of the developed system. During the test, the system was operated stably, and temperature did not exceed over $48^{\circ} \mathrm{C}$ at power amplifier and $54^{\circ} \mathrm{C}$ for air compressor. The dimension of the actuator was $18 \times 18 \times 45 \mathrm{~mm}^{3}(\mathrm{~W} \times \mathrm{L} \times \mathrm{H})$, and the weight was approximately 47 grams. The stimulation area was circular and $16 \mathrm{~mm}$ in diameter.
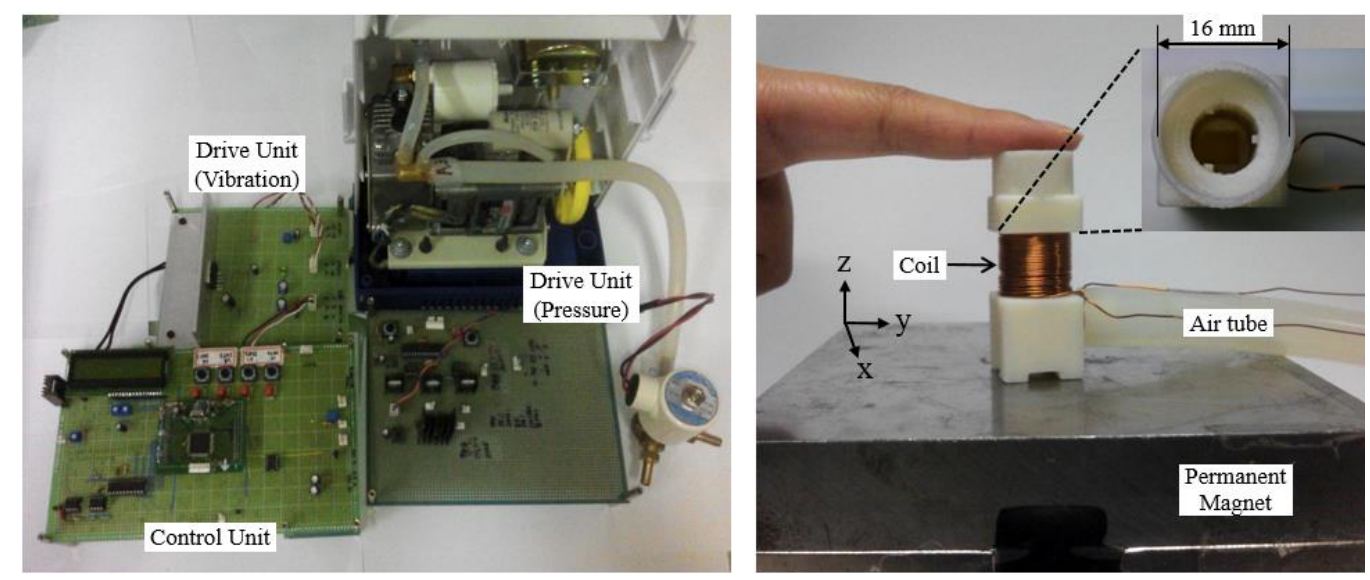

Fig. 4. Photographs of the developed vibration- and pressure-stimulation system. 
Figure 5 shows the raw data from the three-axis accelerometer and pressure sensor when two types of stimulation (vibrotactile + pressure sense) were simultaneously applied. A vibrotactile stimulation of 1.8 gravity at $200 \mathrm{~Hz}$ and pressure sense stimulation with a maximum pressure of 9 psi were displayed. Vibrotactile stimulation followed the sinusoidal waveform transmitted from the control unit. The intensity of $z$-axis is relatively smaller than that of $x$ - and $y$-axis because the direction of the finger pressing force is the same as that of z-axis (Figure 5(a)). It took approximately $0.3 \mathrm{sec}$ for the pressure sense stimulation to reach the maximum pressure of 9 psi and nearly less than $0.1 \mathrm{sec}$ to remove the pressure (Figure 5(b)).

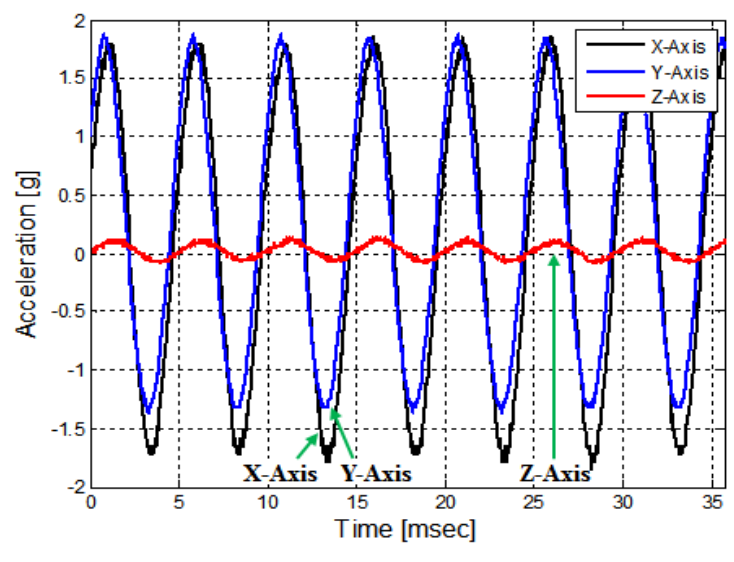

(a)

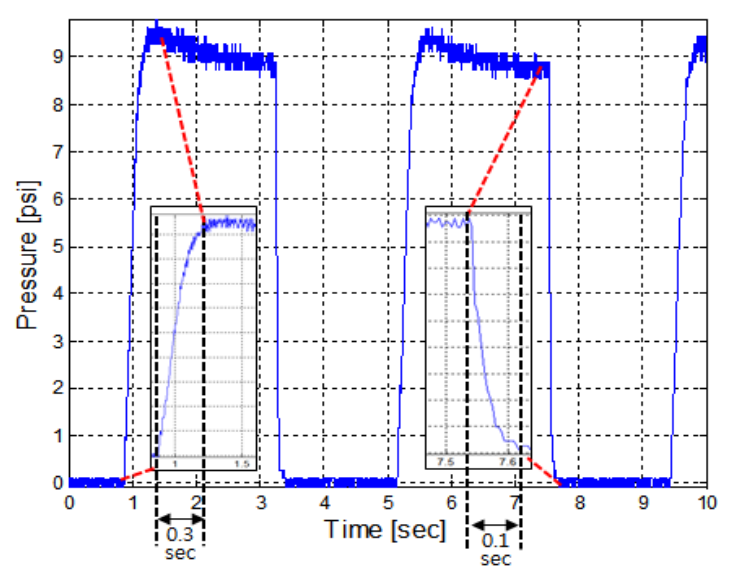

(b)

Fig. 5. Raw data from the three-axis accelerometer (a) and pressure sensor (b).

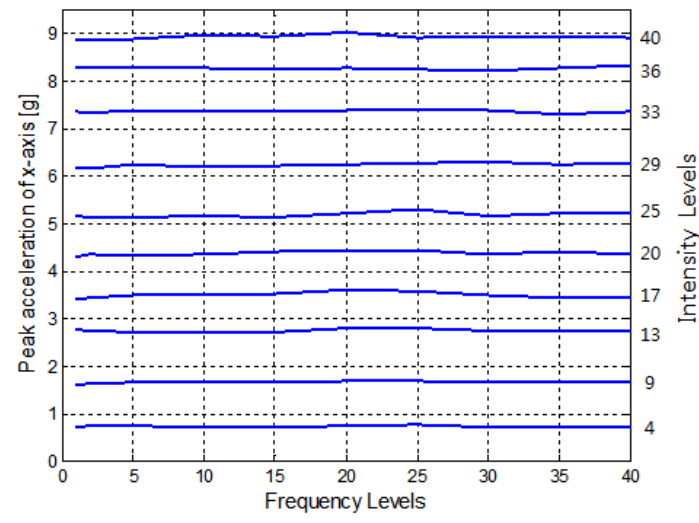

(a)

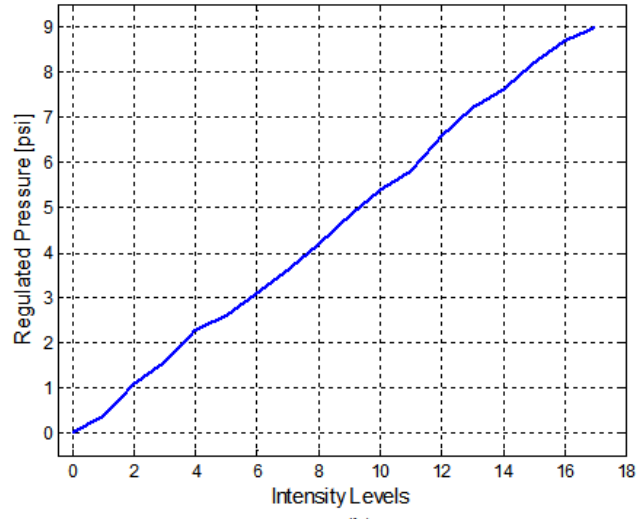

(b)

Fig. 6. The peak acceleration of $\mathrm{x}$-axis with different frequencies and intensities (a) and regulated pressure with different intensities (b). 
Figure 6 shows the response characteristics of the developed system with different stimulation parameters such as frequencies and intensities. The stimulation intensities for vibrotactile stimulation were maintained constantly as the frequency changes (Figure 6(a)). It has a wide stimulation intensities range of 0 to 9.0 gravities with 40 levels and stimulation range of 0 to $400 \mathrm{~Hz}$ comprising 40 levels. The simulation intensity could be varied with distance from the center of the permanent magnet. However, it does not have to be considered because the actuator does not change its position during inter- and intra-experiment. The regulated pressure during pressure-stimulation increased linearly with increased intensity levels (Figure 6(b)). Also, the duration of stimulation can be easily controlled using a micro-controller.

Figure 7 shows the results of the spectrogram and topography analysis of the C3 and C4 areas. These analysis used established methods to express the brain functionality during an event-related desynchronization (ERD) and an event-related synchronization (ERS) $[18,19]$. All three types of stimulation showed an event-related desynchronization that decreased the power within approximately $0.5 \mathrm{sec}$ after stimulation display at the beta band (20 to $30 \mathrm{~Hz})$, followed by subsequent identification of an event-related synchronization increasing the power (Figure 7(a)). This is the typical EEG pattern seen when tactile sensation is perceived [20]. The overall cortical response of the brain can be confirmed from the topography results [17]. The contralateral somatosensory area was largely activated for all three types of stimulation (Figure 7(b)).

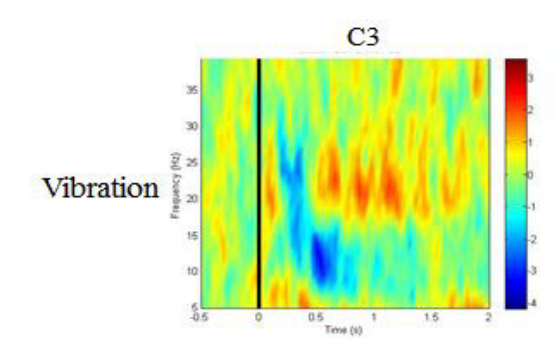

C3

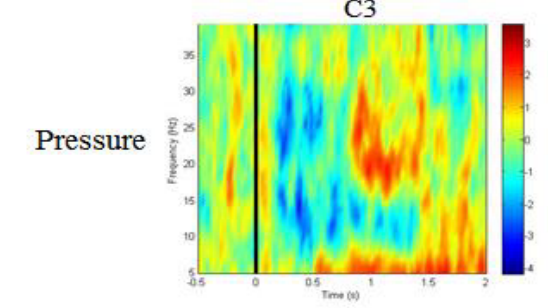

C3

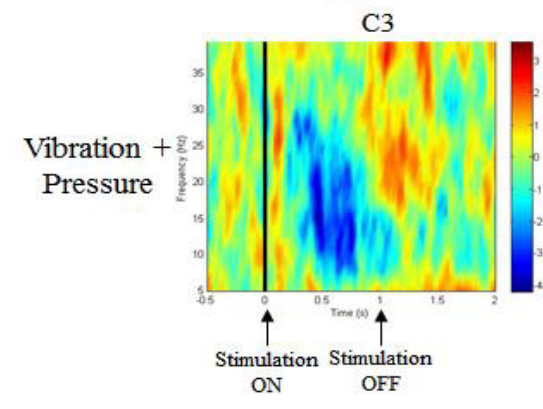

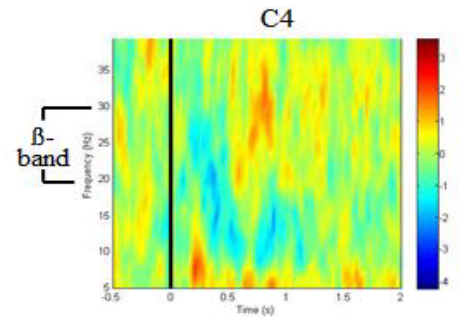

C4

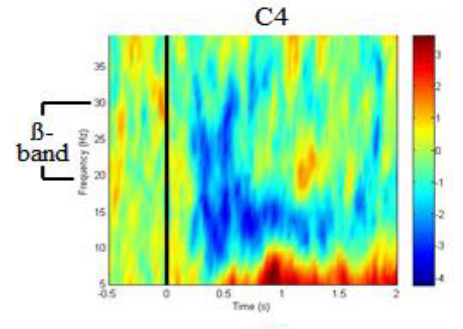

C4

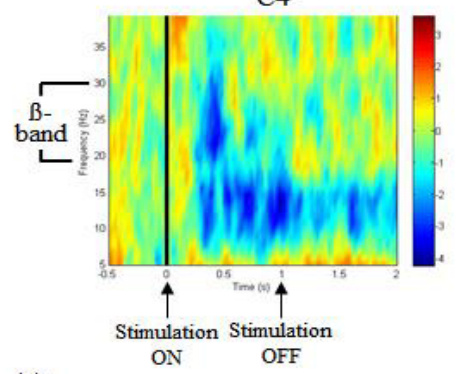

(a)
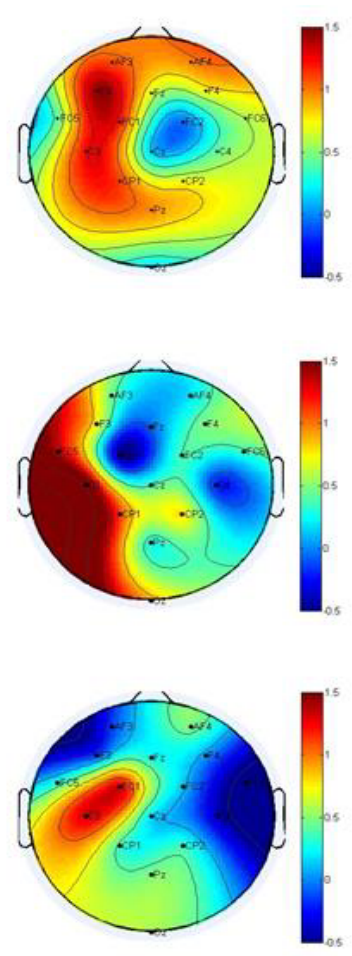

(b)

Fig. 7. EEG spectrum (a) and topography (b) for the three types of tactile stimulation. The brain topography was captured at $0.8 \mathrm{sec}$. 


\section{Discussion}

In this study, a tactile stimulation system was developed that can display separate and simultaneous tactile sensations of vibrotactile and pressure senses for complex tactile cognitive studies. The developed system consisted of a control unit, a drive unit, and an actuator. The overall system is affordable, easy to use and can be operated with a PC or manually.

Tactile sensation has high variability in cognition and perception characteristics according to the stimulation frequency, intensity, and duration, area, etc. Therefore, the stimulation parameters should be controlled quantitatively. From our test run, it was verified that this system could quantitatively control the stimulation parameters of vibrotactile and pressure sense. To check whether this system could be utilized as a tactile stimulator, EEG signals for three types of tactile stimulation, namely, vibrotactile $(\mathrm{V})$, pressure sense $(\mathrm{P})$, and simultaneous vibrotactile + pressure sense stimulation $(\mathrm{V}+\mathrm{P})$ were analyzed. All three types of stimulation induced a change in the ERD and ERS responses in the $\mathrm{C} 3$ and $\mathrm{C} 4$ areas, suggesting that the tactile sensation was correctly perceived using the developed tactile stimulator. The topography map showed that different cortical activations and clear responses were identified in the contralateral somatosensory area for all three types of stimulations. For future studies, a specific study on the difference of the three types of stimulations including complex tactile sensation (vibrotactile + pressure sense) through detailed experimental design and EEG signal analysis can be performed.

We anticipate that the developed tactile stimulator system can be actively utilized for various kinds of complex tactile studies. This is because our system can control the stimulation parameters quantitatively and display two types of tactile sensations separately or simultaneously with one actuator without changing the stimulation system.

\section{Acknowledgement}

This research was supported by the Pioneer Research Center Program (2011-0027920), and Midcareer Researcher Program (NRF-2012R1A2A2A01007998) through the National Research Foundation of Korea funded by the Ministry of Science, ICT \& Future Planning. This research was also partially supported by Basic Science Research Program through the National Research Foundation of Korea (NRF) funded by the Ministry of Education (NRF-2013R1A1A2005299).

\section{References}

[1] G.S. Harrington, C.T. Wright and J.H. Downs III, A new vibrotactile stimulator for functional MRI, Human Brain Mapping 10 (2000), 140-145.

[2] V. Jousmaki, N. Nishitani and R. Hari, A brush stimulator for functional brain imaging, Clinical Neurophysiology 118 (2007), 2620-2624.

[3] C. Dresel, A. Parzinger, C. Rimpau, C. Zimmer, A.O. Ceballos-Baumann and B. Haslinger, A new device for tactile stimulation during fMRI, Neuroimage 39 (2008), 1094-1103.

[4] R.W. Dykes, A. Miquee, C. Xerri, Y. Zennou-Azogui, C. Rainville, A. Dumoulin and D. Marineau, A delivery device for presentation of tactile stimuli during functional magnetic resonance imaging, Journal of Neuroscience Methods 159 (2007), 308-317.

[5] L. Yao, J. Meng, D. Zhang, X. Sheng and X. Zhu, Selective sensation based brain-computer interface via mechanical vibrotactile stimulation, Plos One 8 (2013), e64784. 
[6] Y. Ke, L. Chen, L. Fu, Y. Jia, P. Li, X. Zhao, H. Qi, P. Zhou, L. Zhang, B. Wan and D. Ming, Visual attention recognition based on nonlinear dynamical parameters of EEG, Bio-Medical Materials and Engineering 24 (2014), 349355

[7] H.S. Kim, M.H. Choi, S.P. Hong, J.C. Lee, S.J. Park, U.H. Jeong, S.C. Chung and J.H. Yi, A simple finger stimulator for simultaneous vibration and heat stimulation, Applied Mechanics and Materials 513 (2014), 3407-3410.

[8] D.W. Kim, K.W. Seong, M.N. Kim, J.H. Cho and J.H. Lee, A 1-channel 3-band wide dynamic range compression chip for vibration transducer of implantable hearing aids, Bio-Medical Materials and Engineering 24 (2014), 1009-1017

[9] E.R. Gizewski, O. Koeze, K. Uffmann, A. de-Greiff, M.E. Ladd and M. Forsting, Cerebral activation using a MRcompatible piezoelectric actuator with adjustable vibration frequencies and in vivo wave propagation control, Neuroimage 24 (2005), 723-730.

[10] M.M. Chakravarty, S. Broadbent, P. Rosa-Neto, C.M. Lambert and D.L. Collins, Design, construction, and validation of an MRI-compatible vibrotactile stimulator intended for clinical use, Journal of Neuroscience Methods 184 (2009), $129-135$.

[11] E. Gallasch, M. Fend, D. Rafolt, R. Nardone, A. Kunz, M. Kronbichler, R. Beisteiner and S. Golaszewski, Cuff-type pneumatic stimulator for studying somatosensory evoked responses with fMRI, Neuroimage 50 (2010), 1067-1073.

[12] M. Montant, P. Romaiquere and J.O. Roll, A new vibrator to stimulate muscle proprioceptors in fMRI, Human Brain Mapping 30 (2009), 990-997.

[13] S.J. Graham, W.R. Staines, A. Nelson, D.B. Plewes and W.E. Mcllory, New devices to deliver somatosensory stimuli during functional MRI, Magnetic Resonance in Medicine 46 (2001), 436-442.

[14] R.S. Huang and M.I. Sereno, Dodecapus: An MR-compatible system for somatosensory stimulation, Neuroimage 34 (2007), 1060-1073.

[15] S.A. Overduin and P. Servos, Distributed digit somatotopy in primary somatosensory cortex, Neuroimage 23 (2004), $462-472$.

[16] A. Gallace, H.Z. Tan, P. Haggard and C, Spence, Short term memory for tactile stimuli, Brain Research 1190 (2007), $132-142$.

[17] C.J. McAllister, K.C. Ronnqvist, I.M. Stanford, G.L. Woodhall, P.L. Furlong and S.D. Hall, Oscillatory beta activity mediates neuroplastic effects of motor cortex stimulation in humans, The Journal of Neuroscience 33 (2013), 79197927.

[18] G. Pfurtscheller and C. Neuper, Event-related synchronization of mu rhythm in the EEG over the cortical hand area in man, Neuroscience Letters 174 (1994), 93-96.

[19] M. Zaepffel, R. Trachel, B.E. Kilavik and T. Vrochier, Modulations of EEG beta power during planning and execution of grasping movements, Plos One 8 (2013), e60060.

[20] T. Bardouille, T.W. Picton and B. Ross, Attention modulates beta oscillation during prolonged tactile stimulation, European Journal of Neuroscience 31 (2010), 761-769. 